\title{
Multiparametric MRI diagnostic value in a case of prostate cancer
}

\author{
Gelu Adrian Popa ${ }^{1,4}$, loana Gabriela Lupescu ${ }^{1,4}$, Emi M. Preda ${ }^{1,4}$, Cristina Nicolae ${ }^{1}$, Anca Filip Flintoaca ${ }^{1}$, \\ Rãzvan Alexandru Capșa ${ }^{1,4}$, C. Scheau ${ }^{1,4}$, A. Dijmarescu ${ }^{1,4}$, Mihaela Mihai ${ }^{2}$, A. Preda ${ }^{3}$
}

${ }^{1}$ Radiology, Medical Imaging and Interventional Radiology Department, Fundeni Clinical Institute, Bucharest, Romania

2Department of Histopathology, Fundeni Clinical Institute, Bucharest, Romania

${ }^{3}$ Center for Uronephrology and Renal Transplantion, Fundeni Clinical Institute, Bucharest,

Romania

${ }^{4}$ University of Medicine and Pharmacy «Carol Davila», Bucharest, Romania
Corresponding author:

Ioana G.Lupescu and Popa Gelu-Adrian Radiology and Medical Imaging Department, Fundeni Clinical Institute 258 Fundeni Str., Sector 2, 022328 Bucharest, Romania

E-mail: ilupescu@gmail.com geluadrianpopa@yahoo.com

\section{ABSTRACT}

Prostate cancer (PC) is one of the most prevalent malignant tumour in men, especially in elderly patients. The aim of this paper is to present and illustrate the utility of multiparametric (MP) MRI in the diagnostic of PC. A 66 years old patient with clincal suspicion of PC was explored by MP MRI. The MR examination included T2-wi, dynamic contrast-enhanced (DCE) MRI and diffusion-weighted imaging (DWI) acquisitions. The final MR diagnosis was prostate tumor with the TNM-stage T4 N1 Mx. Radical prostatectomy revealed a Gleason score $7(4+3)$ adenocarcinoma with extraprostatic extension. This case report shows the utility of MP MRI exam in characterizing and for the management of $\mathrm{PC}$.

Key words: prostate cancer, MP MRI, ADC map, DCE

\section{OBJECTIVES}

The aim of this paper is to present and illustrate the usefulness of MP MRI exam in the detection, localization and staging of prostate cancer in a 66 years old male patient.

\section{CASE REPORT}

The patient was admitted with an elevated PSA level of 37,5 ng/ml and clinical suspicion of PC. His past medical history were non-contributory.

MP MRI prostate examination was performed using a $1.5 \mathrm{~T}$ superconducting MR system with a phased array surface coil. An anti-peristaltic drug was injected intravenously before the examination in purpose to reduce peristaltic movement of the ileum and sigmoid colon. MP MRI examination was performed using T2-wi, dynamic contrast-enhanced (DCE) MRI and diffusion-weighted imaging (DWI) using multiple b factor: 0, 1000 and $1500 \mathrm{~s} / \mathrm{mm}^{2}$. For optimal 
prostate characterization we use the same thickness and spacing in the axial plane, as well as sections perpendicular to the rectal wall or positioned in the plane of the seminal vesicles and ejaculatory ducts. The extracellular gadolinium-based contrast agent was intravenously injected $(0.1 \mathrm{mmol} / \mathrm{kg}$ of body weight) followed by a $20 \mathrm{ml}$ saline flush. The image acquisition parameters are summarized in table 1 (1).

For the MR imaging analysis we have used PI-RADS scoring system proposed by the European Society of Urogenital Radiology (ESUR) in 2012 (2). The PI-RADS scoring system uses a five-point scale $(2,3)$ :

- Score 1: clinically significant disease is highly unlikely to be present;

- Score 2: clinically significant cancer is unlikely to be present;

- Score 3: clinically significant cancer is equivocal;

- Score 4: clinically significant cancer is likely to be present;

- Score 5: clinically significant cancer is highly likely to be present.

The day after the MRI examination was performed a prostate biopsy, and 7 weeks later, the patient underwent radical prostatectomy.

\section{RESULTS}

\section{MP MRI}

T2-w images shows a few areas of low signal intensity with irregular shape and ill-defined contours located in the base and mid part of the prostate gland involving the right peripheral zone (PZ) and the transition zone (TZ).

DWI and (apparent diffusion coeficient) ADC map shows restricted diffusion in these areas appearing with high signal intensity on DWI, respectively low signal intensity on the ADC map. The ADC values of prostate cancer and non-cancerous peripheral and transitional tissue at $b=0-1000$ and $0-1500 \mathrm{~s} / \mathrm{mm}^{2}$ are summarized in table 2.

DCE MRI shows a type 2 of time intensity-curve (gadolinium concentration) representing a plateau enhancement after initial uptake.

The MRI findings are suggestive for a prostatic tumor (figure 1).

The right seminal vesicle appears in low signal intensity on T2-w images with restricted diffusion and type 2 of time intensity-curve (plateau), associating thickening of the ductus deferens and obliteration of

Table 1 - Procedure details (1)

\begin{tabular}{|c|c|c|c|c|c|c|c|}
\hline Sequences & type & $\begin{array}{c}\mathrm{TR} \\
(\mathrm{ms})\end{array}$ & $\begin{array}{c}\mathrm{TE} \\
(\mathrm{ms})\end{array}$ & NAQ & $\begin{array}{l}\text { Thickness/Spacing } \\
\text { (mm) }\end{array}$ & Matrix & $\begin{array}{l}\text { FOV } \\
(\mathrm{cm})\end{array}$ \\
\hline \multirow[t]{2}{*}{ T2-wi sagittal } & FSE JET & 5907 & 120 & 1 & $4 / 0.4$ & $272 \times 272$ & $24 \times 24$ \\
\hline & cover the entire pelvis & & & & & & \\
\hline \multirow[t]{2}{*}{ T2-wi axial } & FSE & 4876 & 120 & 2 & $4 / 0.4$ & $352 \times 352$ & $32.2 \times 26.9$ \\
\hline & cover the entire prostate & & & & & & \\
\hline \multirow[t]{2}{*}{ T2-wi oblique } & FSE & 4389 & 120 & 4 & $4 / 0.4$ & $352 \times 352$ & $24 \times 24$ \\
\hline & $\begin{array}{l}\text { pover the entire prostate with sections } \\
\text { respectively in the plane of the seminal } \\
\text { vesicles and ejaculatory ducts }\end{array}$ & & & & & & \\
\hline \multirow[t]{2}{*}{ STIR coronal } & FSE & 3950 & 48 & 1 & $4 / 1$ & $320 \times 320$ & $38 \times 30$ \\
\hline & $\begin{array}{l}\text { cover the entire pelvis, optional seguence } \\
\text { when we evidentiated bone lesions }\end{array}$ & & & & & & \\
\hline \multirow[t]{2}{*}{ T1-wi axial } & SE & 625 & 15 & 1 & $4 / 0.4$ & $352 \times 224$ & $32.2 \times 26.9$ \\
\hline & cover the entire prostate & & & & & & \\
\hline \multirow[t]{2}{*}{ DWl axial } & SE EPI & 4218 & 84 & 9 & $4 / 0.4$ & $144 \times 144$ & $28 / 28$ \\
\hline & $\begin{array}{l}\text { cover the entire prostate with } b \text { values of } 0 \text {, } \\
1000 \text { and } 1500 \mathrm{~s} / \mathrm{mm}^{2}\end{array}$ & & & & & & \\
\hline \multirow[t]{2}{*}{$\begin{array}{l}\text { MR-dynamic } \\
\text { contrast enhanced } \\
\text { (DCE MRI) }\end{array}$} & 3D FFE (Fast Field Echo) FatSat Dynamic & 4.8 & 1.9 & 1 & $\begin{array}{c}4 / 0 \\
\text { interpolated } \\
\text { to } 2 / 0\end{array}$ & $176 \times 180$ & $23 \times 23$ \\
\hline & $\begin{array}{l}\text { cover the entire prostate in axial plane; } \\
\text { duration } 10 \text { seconds per cover, repeated } \\
\text { for } 5-10 \text { minutes continous scan }\end{array}$ & & & & & & \\
\hline \multirow[t]{2}{*}{ T1-wi late phase } & 3D FFE Flow Compensation Isotropic Fat Sat & 30 & 5.5 & 1 & $0.6 / 0$ & $240 \times 240$ & $30 \times 30$ \\
\hline & cover the entire prostate & & & & & & \\
\hline
\end{tabular}


Table 2 - The ADC values of cancerous and non-cancerous tissue at $b=0-1000$ and $0-1500 \mathrm{~s} / \mathrm{mm}^{2}$

\begin{tabular}{lccc}
\hline b value $\left(\mathbf{s} / \mathbf{m m}^{2}\right)$ & \multicolumn{3}{c}{ ADC values \pm standard deviation $\left(\mathbf{X} 10^{-3} \mathbf{~ m m}^{2} \mathbf{s}\right)$} \\
\hline & Prostate cancer & Non-cancerous tissue \\
\hline & & PZ & TZ \\
\hline $\mathrm{b}=0-1000$ & $0.89 \pm 0.11$ & $1.65 \pm 0.16$ & $1.29 \pm 0.20$ \\
$\mathrm{~b}=0-1500$ & $0.81 \pm 0.15$ & $1.57 \pm 0.13$ & $1.31 \pm 0.14$ \\
\hline
\end{tabular}

the angle between the prostate and seminal vesicle; the MRI findings are suggestive for a tumour extension into the right seminal vesicle (figure 2).

Furthermore, the prostatic tumor is located adjacent to the bladder neck with loss of low T2 signal in bladder muscle and abnormal enhancement; the MRI findings are suggestive for bladder neck involvement (figure 3).

In this case report, lesion was classified according to the PI-RADS scoring system as follows:

- T2-wi, PI-RADS 5 (invasive behaviour - right seminal vesicles and bladder neck);

- DWI/ADC map, PI-RADS 5 (high signal intensity on the high b-value DWI with low signal intensity on ADC map);

- DCE MRI PI-RADS 3 (type 2 gadolinium enhance- ment curve and enhancement at an unusual place).

The MRI exam also revealed a right iliac tumoral adenopathy (figure 4).

There was no evidence of bone lesions on coronal STIR acquisition covering the entire pelvis.

In this case the final diagnosis was prostate tumor with the following TNM-stage T4 N1 Mx.

\section{Histological findings}

The biopsy of prostate confirmed the diagnosis of prostate cancer with Gleason score $7(3+4)$ on the right side, respectively $7(4+3)$ on the left side.

Radical prostatectomy revealed a Gleason score 7 $(4+3)$ adenocarcinoma with extraprostatic extension (figure 5).

\section{DISCUSSION}

The zonal anatomy of prostate can be easily characterized using T2-w images $(1,4)$. In older male patients, the transition zone increase in size due to benign prostatic hypertrophy (BPH) and the central zone is compressed and most often difficult to identify on MR images (5).
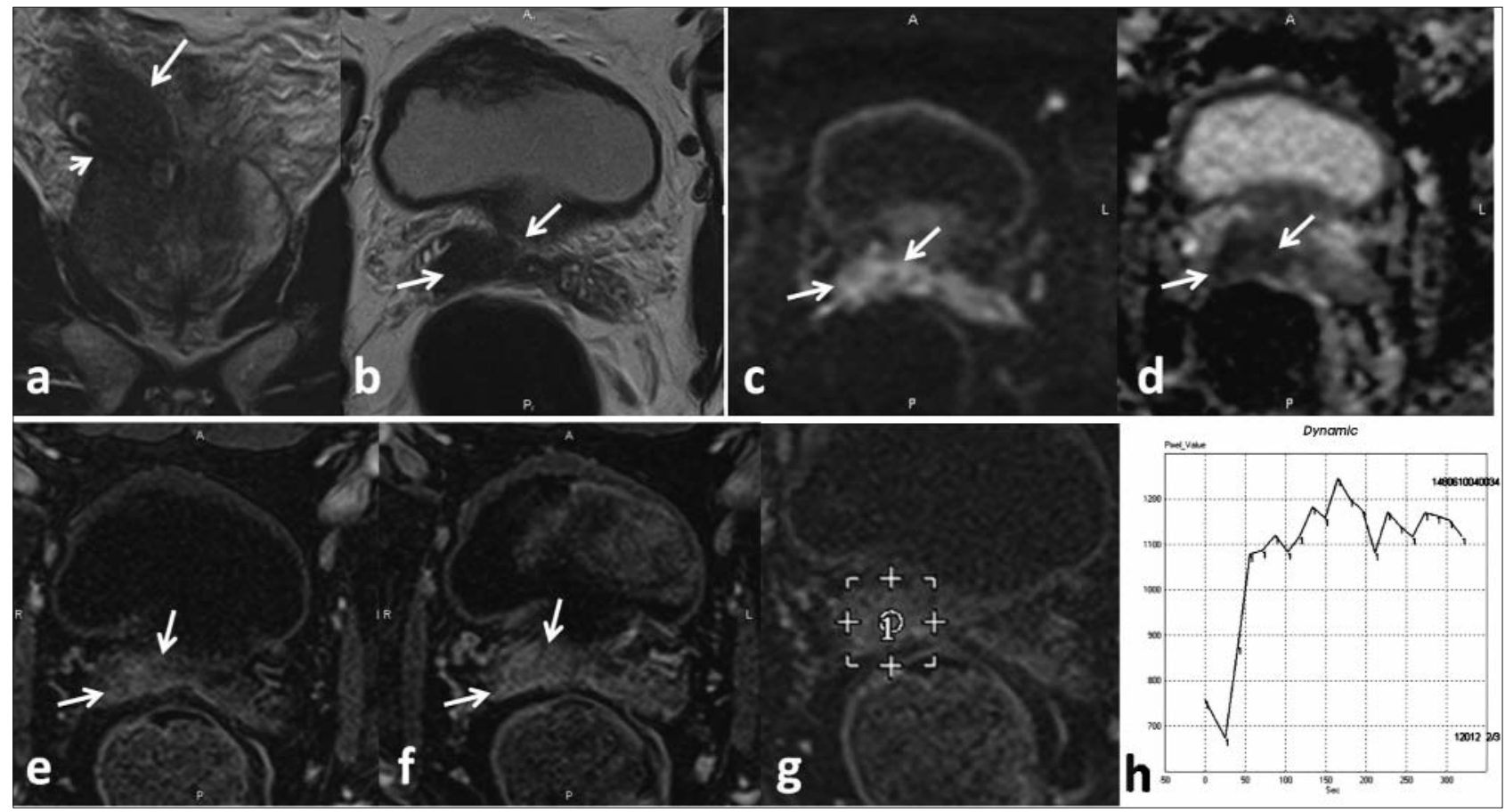

Figure 1 - Prostate cancer / MP MRI - T2-wi (a), DWI with b value of $1000 \mathrm{~s} / \mathrm{mm} 2$ and ADC map (b, c), DCE-MRI and time-intensity (gadolinium concentration) curve (d-early phase, e-late phase, f-ROI in tumoral mass and g-the corresponding graph). Tumoral mass (arrows) located in the prostate gland involving the right PZ and the TZ with irregular shape and ill-defined contours; this lesion appear hypointense in T2-wi (a), with restricted diffusion (b and c) and early, rapid and persistent enhancement / type 2 of the dynamic curve (plateau) (d-g) 


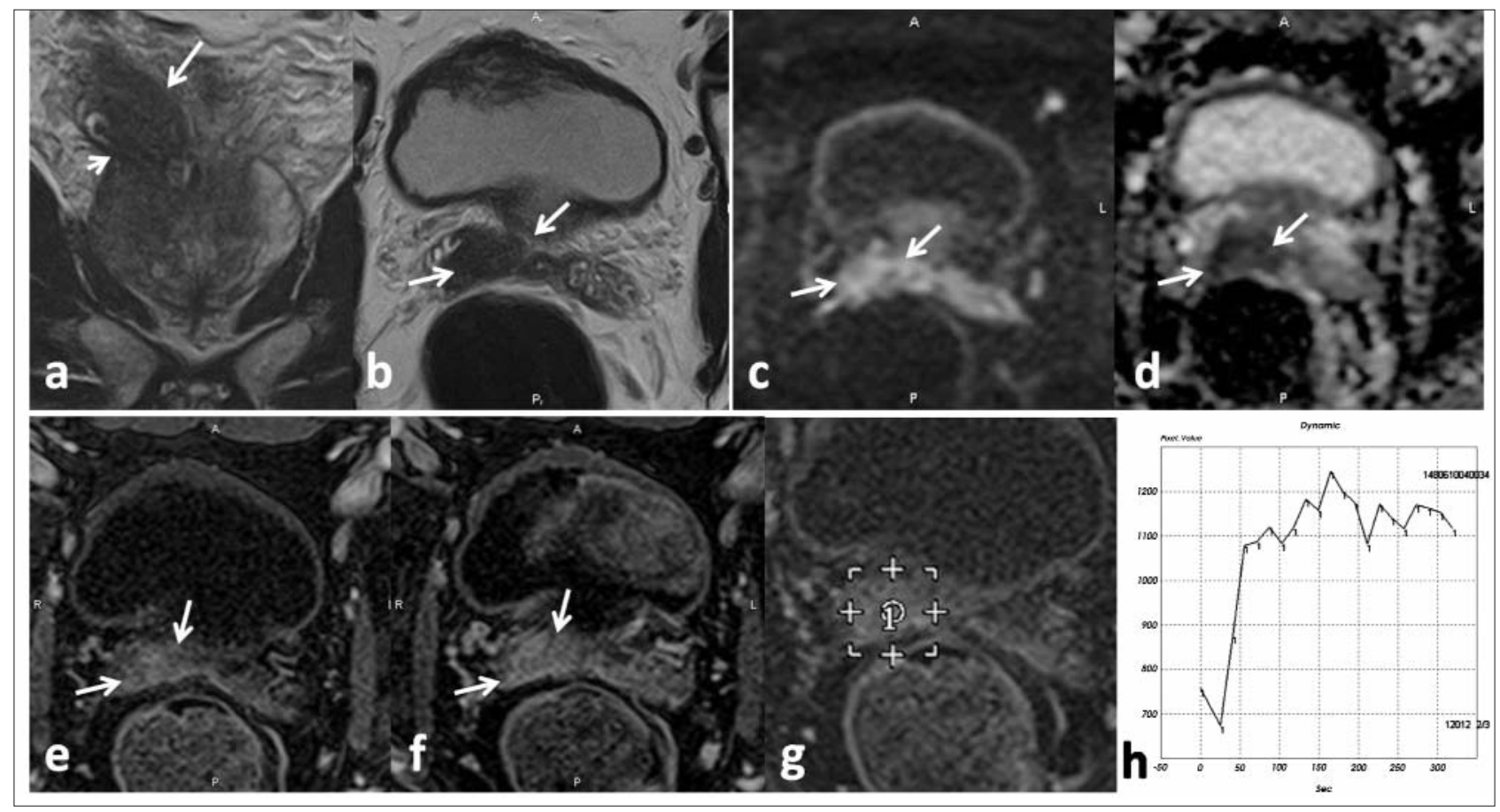

Figure 2 - The right seminal vesicle invasion / MP MRI - T2-wi (a-coronal and b-axial), DWI with b value of $1000 \mathrm{~s} / \mathrm{mm}^{2}$ and ADC map (c, d), DCE-MRI and time-intensity (gadolinium concentration) curve (e-early phase, f-late phase, $g$-ROI in the right seminal vesicle and h-the corresponding graph). The right seminal vesicle (arrows) appears in low signal intensity on T2-w images (a and b) with restricted diffusion ( $c$ and d) and type 2 of time intensity-curve (plateau) (e-h), associating obliteration of the angle between the prostate and seminal vesicle (arrowhead)

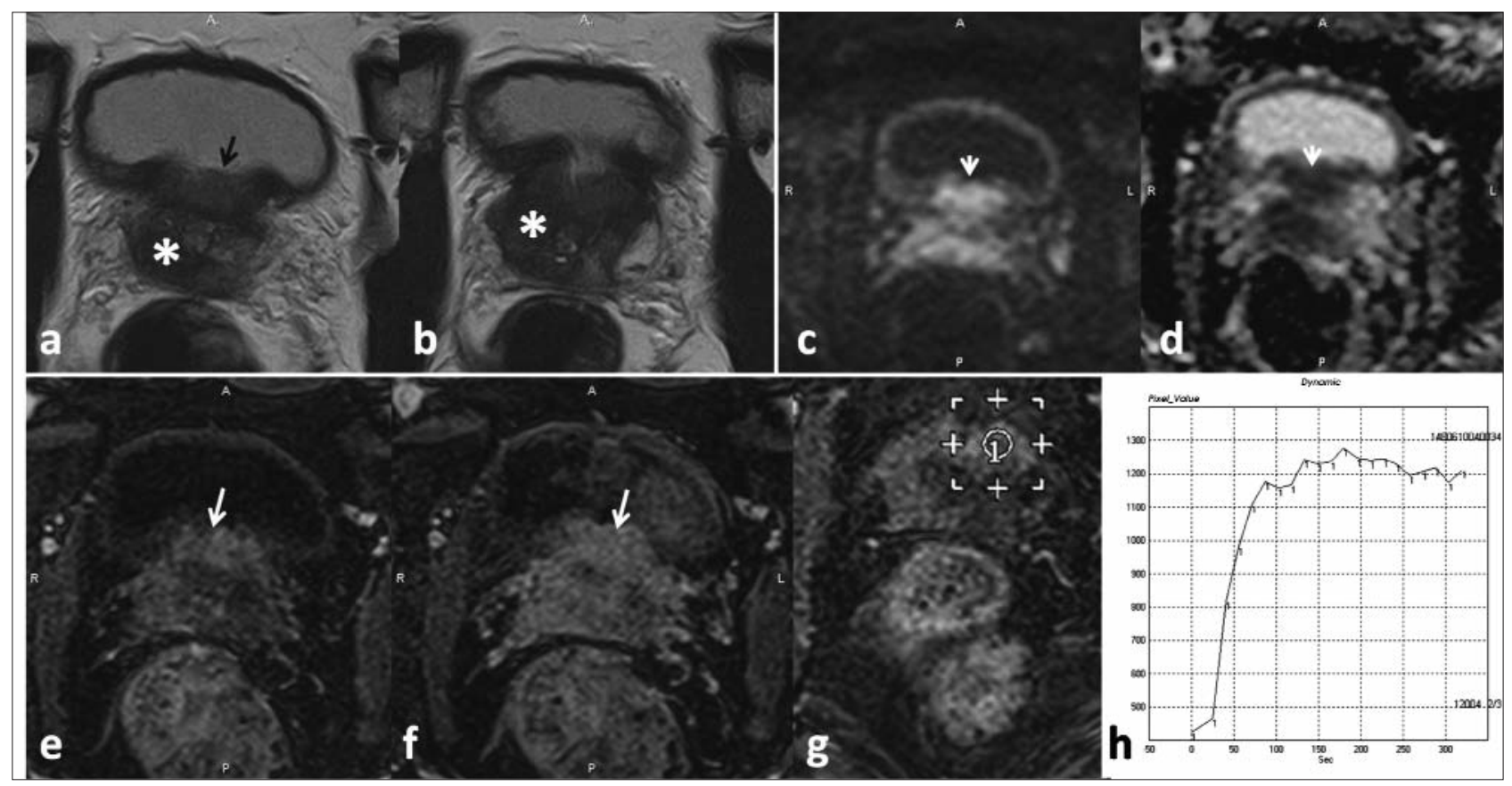

Figure 3 - The bladder neck involvement / MP MRI - T2-wi (a and b), DWI with b value of $1000 \mathrm{~s} / \mathrm{mm} 2$ and ADC map (c, d), DCE-MRI and time-intensity (gadolinium concentration) curve (e-early phase, f-late phase, $g$-ROI located adjacent to the bladder neck and h-the corresponding graph). The prostatic tumor (stars) is located adjacent to the bladder neck, with loss of low T2 signal in bladder muscle (black arrow), restricted diffusion (arrowheads) and abnormal enhancement (white arrows) 


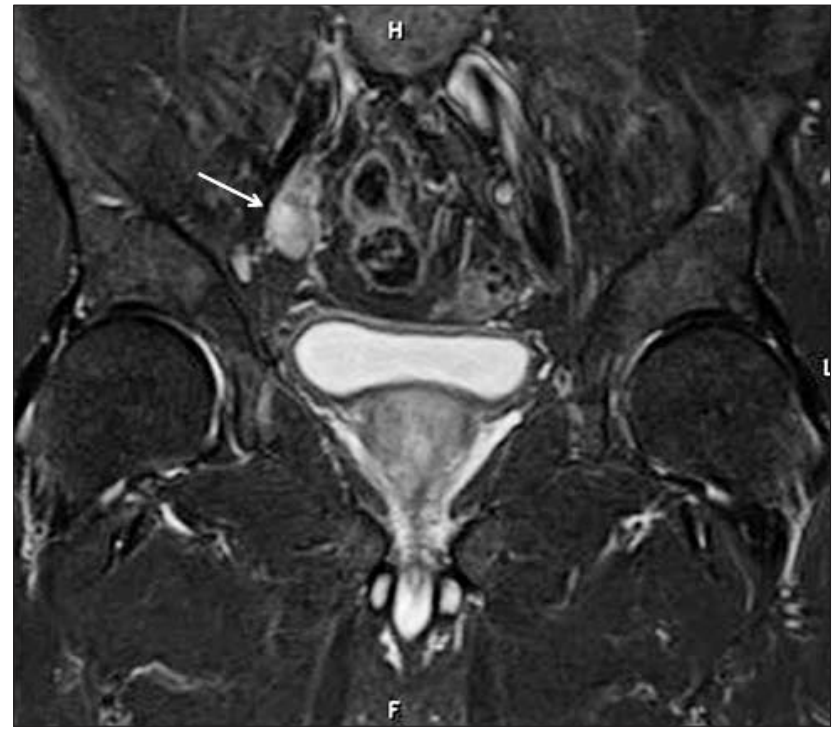

Figure 4 - Right iliac tumoral adenopathy (arrow) (coronal STIR)
In prostatic hyperplasia, DCE MRI and DWI may reveal variably abnormal findings, such that the T2-wi become more accurate in differentiating suspicious areas from benign lesions (6).

Nicolas Barry Delongchamps et al. reported that the combination of DWI and DCE MRI to T2-wi, significantly increase diagnostic performance in cancer detection in the PZ, as well as Gleason score, but fails in the TZ (7); the sensitivity of T2-wi alone, T2-wi+DCE, T2-wi+DWI and T2-wi+DCE+DWI, for the PZ were 63\%, 79\%, 81\% and $80 \%$, respectively, and for the TZ were $71 \%, 47 \%$, $71 \%$ and $53 \%$, respectively (7); the specificity of T2-wi alone, T2-wi+DCE, T2-wi+DWI and T2-wi+DCE+DWI, for the $\mathrm{PZ}$ were $98 \%, 92 \%, 93 \%$ and $97 \%$, respectively, and for the TZ were $98 \%, 77 \%, 98 \%$ and $83 \%$, respectively (7).

Brian Kim et al. analyzed the diagnostic accuracy of
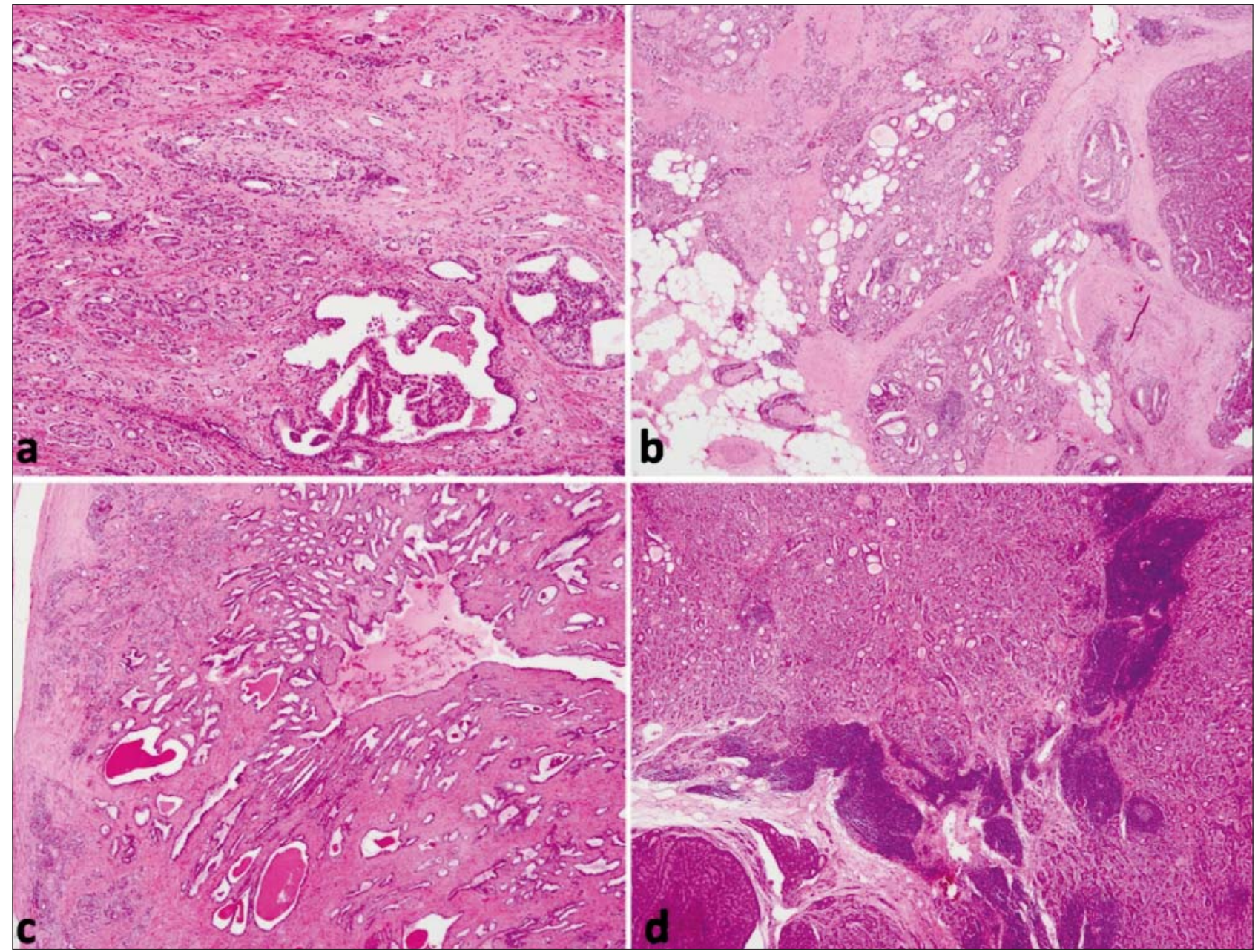

Figure 5 - Histological findings - Gleason score $7(4+3)$ acinar adenocarcinoma with extraprostatic extension and tumoral lymphadenopathy; H\&E 10x (a) and 5x (b-d); a - perineural invasion; $b$ - extracapsular extension in periprostatic fibroadipos tissue with perineural invasion; $c$ - seminal vesicle invasion; $d$ - lymphatic metastasis 
surface coil magnetic resonance imaging at $1.5 \mathrm{~T}$ for local staging of prostate cancer in patients with elevated risk (8); the sensitivity and specificity in tumor detection for patients with high risk were $94.6 \%$ and $71.4 \%$, respectively and for patients with intermediate risk were $93.8 \%$ and $100 \%$, respectively (8); regarding detection of extraprostatic extension, the sensitivity and specificity for patients with high risk were $92.3 \%$ and $93.1 \%$, respectively, and for patients with intermediate risk were $50 \%$ and $77.8 \%$, respectively (8); concerning detection of seminal vesicle tumoral invasion, the sensitivity and specificity for patients with high risk were $88.9 \%$ and $88.6 \%$, respectively, and for patients with intermediate risk were $66.7 \%$ and $100 \%$, respectively (8).

The differential diagnosis of prostate cancer must be performed with:

- BPH which appears as well-defined areas with intermediate signal intensity on T2-wi located in the central gland; this is mainly composed of transition zone which appears increased in size, compressing the central and peripheral zones $(1,3,5,9)$.

- Chronic prostatitis, scars or post-radiation therapy changes which appears as areas of low signal intensity on T2-wi frequently located in the peripheral zone, with triangular or linear shape and without mass $(1,4,5,10)$.

- Blood within prostate after biopsy which leads to high signal intensity on T1-wi and low signal intensity on T2-wi $(1,4,5,11)$.

- Prostatic calcifications which appears as small areas with low signal intensity on both T1-wi and T2-wi sequences (1).

\section{CONCLUSIONS}

This case report shows and illustrate the utility of MP MRI examination in the prostate cancer diagnosis, specifying the role of each MR acquisition. The combination of T2, DWI and DCE MRI increase the diagnostic performance in the detection and extension of $\mathrm{PC}$, but with different sensitivity and specificity for the $\mathrm{PZ}$ and the TZ. The MRI findings provides important data for the management of prostate cancer.

\section{Acknowledgement}

This work received financial support through the project entitled "CERO - Career profile: Romanian Researcher", grant number POSDRU/159/1.5/S/ 135760 , cofinanced by the European Social Fund for Sectoral Operational Programme Human Resources Development 2007-2013.

\section{REFERENCES}

1. Popa G, Scheau C, Preda A et al. - Multiparametric MR Imaging in Prostate Cancer Diagnosis: make it easy, ECR 2015 - EPOS, Vienna - Austria, Poster No.: C-1811, D0I: 10.1594/ecr2015/C-1811.

2. Barentsz J0, Richenberg J, Clements R et al. - ESUR prostate MR guidelines 2012, European Radiology, 2012, vol. 22, no. 4, pp.746-757, D0I 10.1007/s00330-011-2377-y.

3. Bomers JGR and Barentsz JO - Standardization of Multiparametric Prostate MR Imaging Using PI-RADS, Hindawi Publishing Corporation, BioMed Research International, Volume 2014, Article ID 431680, 9 pages, http://dx.doi.org/10.1155/2014/431680.

4. Choi YJ, Kim JK, Kim N et al. - Functional MR Imaging of Prostate Cancer, RadioGraphics 2007; 27:63-77.

5. Hoeks CMA, Barentsz J0, Hambrock T et al. - Prostate Cancer: Multiparametric MR Imaging for Detection, Localization, and Staging, Radiology: Volume 261: Number 1-October 2011, 46-66.

6. Margolis DJA - Multiparametric MRI for Localized Prostate Cancer: Lesion Detection and Staging, Hindawi Publishing Corporation, BioMed Research International, Volume 2014, Article ID 684127, 11 pages, http://dx.doi.org/ $10.1155 / 2014 / 684127$.

7. Delongchamps NB, Rouanne M, Flam T et al. - Multiparametric magnetic resonance imaging for the detection and localization of prostate cancer: combination of T2-weighted, dynamic contrast-enhanced and diffusionweighted imaging, BJU International, 2010, 107, 1411-1418, doi:10.1111/ j.1464-410X.2010.09808.x.

8. Kim B, Breau RH, Papadatos D. Et al. - Diagnostic accuracy of surface coil magnetic resonance imaging at $1.5 \mathrm{~T}$ for local staging of elevated risk prostate cancer, Canadian Urological Association, CUAJ, August 2010, Volume 4, Issue 4, 257-262.

9. Hricak H, Williams RD, Spring DB et al. - Anatomy and pathology of the male pelvis by magnetic resonance imaging, AJR Am J Roentgenol 1983 ; 141 ( 6 ): 1101-1110.

10. Cruz M , Tsuda K, Narumi $Y$ et al . Characterization of low-intensity lesions in the peripheral zone of prostate on pre-biopsy endorectal coil MR imaging, Eur Radiol 2002; 12 ( 2 ): 357- 365, D0l 10.1007/s003300101044.

11. White $\mathrm{S}$, Hricak $\mathrm{H}$, Forstner $\mathrm{R}$ et al. Prostate cancer: effect of postbiopsy hemorrhage on interpretation of MR images, Radiology 1995;195:385-390. 